\title{
A case of acute necrotising pancreatitis following the second dose of Pfizer-BioNTech COVID-19 mRNA vaccine
}

\author{
Talia Walter ${ }^{1}$, Saxon Connor ${ }^{1}$, Catherine Stedman ${ }^{1}$, and Matthew Doogue ${ }^{2}$ \\ ${ }^{1}$ Canterbury District Health Board \\ ${ }^{2}$ University of Otago
}

June 25, 2021

\section{A case of acute necrotising pancreatitis following the second dose of Pfizer-BioNTech COVID- 19 mRNA vaccine}

As of 22 June 2021, there have been about 400,000,000 doses of the Pfizer-BioNTech COVID-19 mRNA vaccine administered world-wide. At this time, 176 cases of pancreatitis have been reported in pharmacovigilance reports submitted to the WHO. ${ }^{1}$ No epidemiological link between the Pfizer-BioNTech COVID-19 mRNA vaccine and pancreatitis has been reported. We report a case of acute necrotising pancreatitis following the second dose of the Pfizer-BioNTech COVID-19 mRNA vaccine. This case has been reported to the New Zealand Pharmacovigilance Centre and the patient has consented to publication of the case report.

A 43-year-old, NZ European male was diagnosed with acute necrotising pancreatitis 10 hours following the administration of his second Pfizer-BioNTech COVID-19 mRNA vaccine. He has a background of atopy with seasonal rhinitis, eczema and asthma, all of which are 'mild'. He takes no regular medications and takes as needed melatonin $2 \mathrm{mg}$ nocte, cetirizine $10 \mathrm{mg}$ for hay fever, and betamethasone and emollient cream for dermatitis. He has no family history of note and works in a senior professional role. He drinks six standard units of alcohol per week, has never been a tobacco smoker and does not use recreational drugs - and his employer undertakes regular drug testing.

He had a previous episode of pancreatitis in 2011, precipitated by high alcohol intake during a holiday abroad. He is otherwise previously well. There is no family history of note, his father was a smoker and died of lung cancer in his $60 \mathrm{~s}$, his mother is well, he has two siblings and three children all of whom are well. He is not aware of any diseases of note in his extended family.

In May 2021 he received his second dose of Pfizer-BioNTech COVID-19 mRNA vaccine, he was well at that time. Four hours after the dose he had two $330 \mathrm{ml}$ bottles of $5 \%$ alcohol beer with his dinner. Six hours after the dose he became unwell with nausea, epigastric pain, and vomiting. Ten hours after the dose blood tests on admission to hospital included: white cell count $18.5 \times 10^{9} / \mathrm{L}(4-11)$, neutrophils $15.5 \times 10^{9} / \mathrm{L}(1.9$ 7.5), lymphocytes $0.8 \times 10^{9} / \mathrm{L}(1.0-4.0), \mathrm{CRP}<3 \mathrm{mg} / \mathrm{L}(<5)$, lipase $23,750 \mathrm{U} / \mathrm{L}(10-70)$, and triglycerides $3.3 \mathrm{mmol} / \mathrm{L}$. Acute pancreatitis was diagnosed. An ultrasound scan showed pancreatitis without evidence of cholelithiasis. He subsequently deteriorated and a computer tomography (CT) scan on 17 May, day 9 of the illness, showed severe necrotising pancreatitis with collections.

During the admission under a specialist hepatobiliary surgical team, known causes of pancreatitis were excluded including: steroid use, trauma, family history of autoimmune conditions, and infection. He did not have the risk factors of obesity, smoking or heavy alcohol consumption. He had the risk factor of a previous episode of acute pancreatitis. The incidence of pancreatitis in New Zealand Europeans in this age group is approximately 50 per 100,000 per year. ${ }^{2}$ Idiopathic cases represent about $15 \%$ of these. 
Using the Naranjo criteria, the pancreatitis being 'caused' by the Pfizer-BioNTech COVID-19 mRNA vaccine score was 6 'probable'. ${ }^{3}$ Clinical case discussion (including a hepatobiliary surgeon, gastroenterologist and clinical pharmacologist) concluded that while idiopathic pancreatitis could not be excluded, the onset of symptoms and findings were consistent with an acute precipitating event around the time the second vaccine dose was administered.

In addition to the 176 cases reported in VigiBase, searches of PubMed and Google Scholar identified two published case reports of pancreatitis following the Pfizer-BioNTech COVID-19 mRNA vaccine. These were following administration of the first dose of the Pfizer-BioNTech COVID-19 mRNA vaccine. In one case symptoms developed several hours after vaccine administration and the other several days after vaccine administration..$^{4,5}$

Acute pancreatitis is a rare in patients with COVID-19 but characteristic patterns of illness have been reported. ${ }^{6}$ This raises the possibility that an immune response to either the virus or the vaccine could have common sequalae.

This case of acute pancreatitis was temporally associated with the Pfizer-BioNTech COVID-19 mRNA vaccine suggesting a causal link. The characteristics of pancreatitis cases associated with the Pfizer-BioNTech COVID-19 mRNA vaccine should be examined to see if a consistent pattern is present.

1. VigiAccess http://www.vigiaccess.org/ - accessed June 24, 2021.

2. Pendharkar SA, Mathew J, Zhao J, Windsor JA, Exeter J, Petrov MS. Ethnic and geographic variations in the incidence of pancreatitis and post- pancreatitis diabetes mellitus in New Zealand: a nationwide population- based study. 2017;130(1450):14.

3. Naranjo CA, Busto U, Sellers EM, et al. A method for estimating the probability of adverse drug reactions. Clin Pharmacol Ther . 1981;30(2):239-245. doi:10.1038/clpt.1981.154

4. Cieślewicz A, Dudek M, Krela-Kaźmierczak I, Jabłecka A, Lesiak M, Korzeniowska K. Pancreatic Injury after COVID-19 Vaccine-A Case Report. Vaccines . 2021;9(6):576. doi:10.3390/vaccines9060576

5. Parkash O, Sharko A, Farooqi A, Ying GW, Sura P. Acute Pancreatitis: A Possible Side Effect of COVID-19 Vaccine. Cureus . Published online April 28, 2021. doi:10.7759/cureus.14741

6. Bircakova B, Bruha R, Lambert L, Grusova G, Michalek P, Burgetova A. A bimodal pattern of the onset of COVID-19 related acute pancreatitis supports both the cytotoxic and immune-related pathogenesis a systematic review. Scand J Gastroenterol . 2021;56(7):870-873. doi:10.1080/00365521.2021.1922751 